\title{
Using diary to uncover users' personal information management (PIM) behaviours
}

\begin{abstract}
There are various techniques used in HCI to understand usersô personal information management (PIM). behaviours. In our project, we used a diary as a technique to uncover more dumping factors as embedded behaviour that users might have while managing their information on their personal information spaces (PIS). In this paper, we described how we conducted a diary study and highlighted how our diary study affected our data. Later, we suggest how a diary study can better be implemented in a HCI project.
\end{abstract}

Keyword: Diary; Personal information management; Dumping behaviour; Qualitative study 